\title{
Integrated Polypyrrole Flexible Conductors for Biochips and MEMS Applications
}

\author{
Rakefet Ofek Almog, ${ }^{1}$ Hadar Ben-Yoav, ${ }^{2}$ Yelena Sverdlov, ${ }^{1}$ Tsvi Shmilovich, ${ }^{1}$ \\ Slava Krylov, ${ }^{1}$ and Yosi Shacham-Diamand ${ }^{1}$ \\ ${ }^{1}$ Faculty of Engineering, Tel Aviv University, Tel Aviv 69978, Israel
${ }^{2}$ Department of Electrical and Computer Engineering, The Institute for Systems Research, University of Maryland, College Park,
MD 20742, USA
}

Correspondence should be addressed to Yosi Shacham-Diamand, yosish@tauex.tau.ac.il

Received 13 March 2012; Accepted 14 May 2012

Academic Editor: Zeev Zalevsky

Copyright (C) 2012 Rakefet Ofek Almog et al. This is an open access article distributed under the Creative Commons Attribution License, which permits unrestricted use, distribution, and reproduction in any medium, provided the original work is properly cited.

\begin{abstract}
Integrated polypyrrole, a conductive polymer, interconnects on polymeric substrates were microfabricated for flexible sensors and actuators applications. It allows manufacturing of moving polymeric microcomponents suitable, for example, for microoptical-electromechanical (MOEMS) systems or implanted sensors. This generic technology allows producing "all polymer" components where the polymers serve as both the structural and the actuating materials. In this paper we present two possible novel architectures that integrate polypyrrole conductors with other structural polymers: (a) polypyrrole embedded into flexible polydimethylsiloxane (PDMS) matrix forming high aspect ratio electrodes and (b) polypyrrole deposited on planar structures. Self-aligned polypyrrole electropolymerization was developed and demonstrated for conducting polymer lines on either gold or copper seed layers. The electropolymerization process, using cyclic voltammetry from an electrolyte containing the monomer, is described, as well as the devices' characteristics. Finally, we discuss the effect of integrating conducting polymers with metal seed layer, thus enhancing the device durability and reliability.
\end{abstract}

\section{Introduction}

Polymeric materials, both insulators and conductors, offer several interesting characteristics useful for MEMS applications. They are especially attractive for applications in which conventional materials such as silicon, glass, and most metals are too stiff. Using polymers with a lower Young's modulus will allow the development of microsensors, optical components, microfluidics and actuators with reduced stiffness [1-3], allowing larger deformations under lower driving forces. They are also characterized by several advantageous properties [4] such as high elasticity, variety of $3 \mathrm{D}$ structure formations, and potentially low cost for mass production. Polymers are attractive candidates for biosensors and implanted devices since many of the polymers are biocompatible and can be chemically functionalized by modifying the devices surface. Polymers are especially attractive for microactuators since their reduced stiffness allows low operating voltages and less power consumption.
We can classify the polymers based on their electrical properties: conductors, insulators, and semiconductor-like polymers. Most of the polymers are insulators, and there are many available structural polymers with microfabrication compatibility and Young's modulus that can exceed $1 \mathrm{GPa}$ (e.g., polyimide) or below $1 \mathrm{MPa}$ (e.g., PDMS). A special class of polymers, the conjugated organic polymers, is conductive and especially attractive for MEMS, MOEMS, and microsensor applications. Conducting polymers can replace metals or inorganic semiconductors, enabling simpler processing of thin films with a wide range of conductivity. Conducting polymers can be considered as potential alternatives to the metals and semiconductors of connecting wires and conductive channels, which are the active materials in optoelectronics, microelectronics, micro-electromechanical systems (MEMS), and micro-sensors.

Simple actuators built using conducting polymers have already been demonstrated. With the discovery in the $1980 \mathrm{~s}$ that conductive polymers are compatible with many of the 


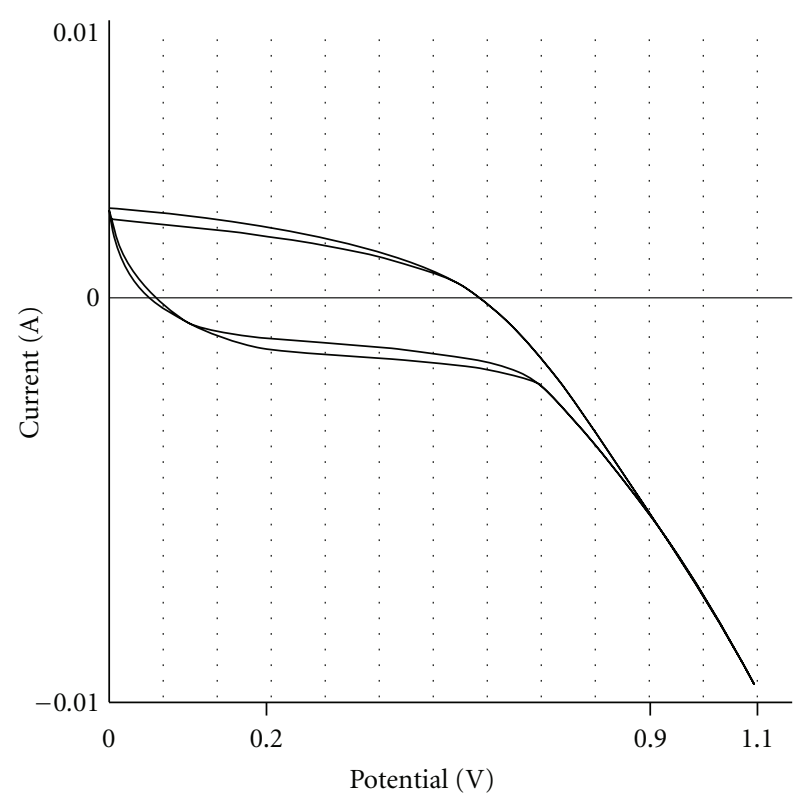

FIGURE 1: CV curves: electro polymerization of pyrrole on thiolmodified Au surface, cycle no. four and five.

biological molecules used in biosensors, research on this subject has expanded greatly [5]. Since there is a need for an "all polymeric" device we investigated the process requirements, devised the process flows, and present here results for a novel polymeric MEMS device built on an organic substrate with conductive polymers. Here we present the deposition process, the patterning, and the electrochemical characterization of the conductive polymers.

In this paper we outline the development of flexible polymeric sensors and actuators. In addition, we describe the fabrication of micron-scale conductive lines and electrodes, embedded into a soft polymeric matrix (e.g., PDMS) [6] or deposited on a flexible polymeric surface. Although metallic electrodes can be used for this purpose, the conducting lines made of polymeric materials exhibit several advantages, including low stiffness, ability to develop high strain, and compatibility with the polymeric environment.

In this work we report the development of a self-aligned electrode patterning process on an organic substrate with conductive polymers. Two configurations were considered, embedded or planar, over a thin metal seed on PDMS. In both cases, selective electrochemical polymerization of polypyrrole was performed. In the first device configuration, the structure consisted of metal (copper) connectors embedded in a flexible PDMS polymer. In the second configuration, its structure consisted of metal (either copper or gold) patterned as MEMS actuator devices. In the embedded device type, the polypyrrole reinforces the copper wires and may act as an electrical shunt over cracks in the metal interconnects, thus increasing the device durability and reliability. In the planar type, the polypyrrole serves as a compliant electrode, allowing large deflections under relatively low bias electrostatic actuation.

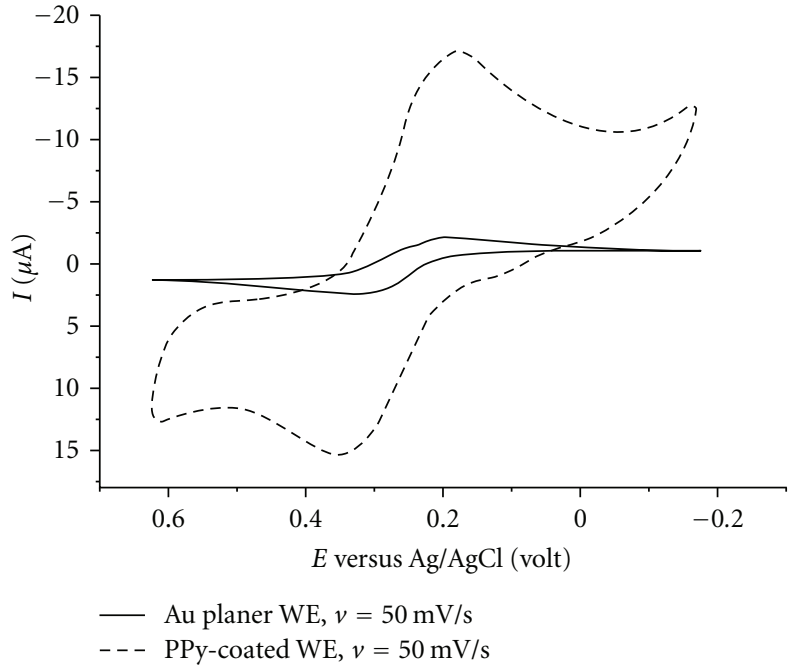

FIgure 2: Cyclic voltammograms resulting from a ferrocyanide/ferricyanide assay with either a PPy-coated (dashed curves) or Au planar (solid curves) working electrode.

\section{Experimental}

The devices were made on planar PDMS structures. We describe here the deposition process, by electropolymerization, of the conducting polymers. The deposition was done by electrochemical polymerization of pyrrole by cyclic voltammetry. An elastic conductive polymer, polypyrrole, was electrochemically polymerized by a CV (cyclic voltammetry) technique using an EG\&G Princeton Applied Research Model 273A potentiostat/galvanostat. In the case of Au-seed the electrochemical polymerization bath contained $0.05 \mathrm{M}_{\text {pyrrole }} \mathrm{C}_{4} \mathrm{H}_{5} \mathrm{~N}$ (Aldrich) and $0.1 \mathrm{M} \mathrm{LiClO}_{4}$ (Aldrich) in acetonitrile $\mathrm{CH}_{3} \mathrm{CN}$ (Sigma-Aldrich). A Pt plate was used as a counter electrode and Calomel ( $\mathrm{KCl}$ saturated) was employed as a reference electrode.

Preselected potential ranges were scanned between 0.0 and $1.2 \mathrm{~V}$ versus SCE reference electrode. The polypyrrole growth is controlled by the number of cycles. The scan rate was $20 \mathrm{mV} / \mathrm{s}$. Electrochemical polymerization (anodic oxidation) of pyrrole by $\mathrm{CV}$ on $\mathrm{Au}$ is illustrated in Figure 1. The polymerization process starts at a potential of $0.6-0.7 \mathrm{~V}$.

Electropolymerization of pyrrole on $\mathrm{Cu}$-seed was performed from aqueous electrolyte, containing $0.1 \mathrm{M}$ sodium salicylate (SS) and $0.5 \mathrm{M}$ polypyrrole. The scan rate was $50 \mathrm{mV} / \mathrm{s}$. Potential ranges were scanned between -0.8 and $+0.8 \mathrm{~V}$ versus Calomel reference electrode. The pyrrole oxidation at a copper electrode, in the presence of SS, starts at about $0.55 \mathrm{~V}$ versus SCE.

The PPy was tested on gold electrodes modified with a thin film of polypyrrole. They were electrochemically characterized and compared to planar gold electrodes. The PPy-modified devices were specifically selected for their relatively high surface area, which was suggested to increase the electrochemical current. 

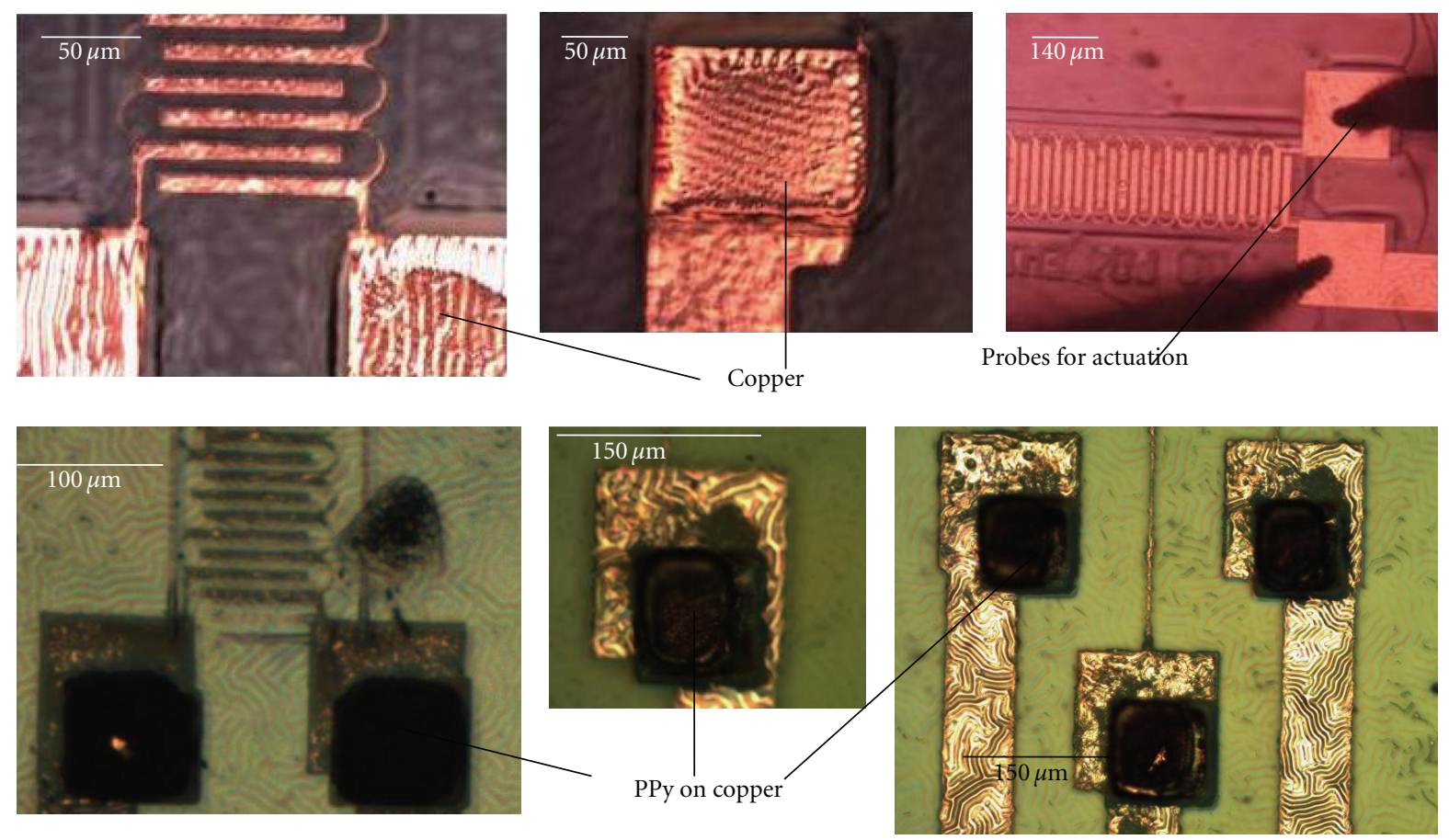

FIgURE 3: Optical micrographs of Polypyrrole electrodeposited on top of copper layer located on a flexible PDMS substrate.

\section{Results}

PPy electrochemical characterization was performed initially. The electrode with and without PPy was evaluated by testing its electrochemical performance for the analysis of a known chemical reaction. Therefore, potassium ferrocyanide $\left(\mathrm{K}_{4}\left[\mathrm{Fe}(\mathrm{CN})_{6}\right], 0.010 \mathrm{M}\right.$, Sigma), potassium ferricyanide $\left(\mathrm{K}_{3}\left[\mathrm{Fe}(\mathrm{CN})_{6}\right], 0.010 \mathrm{M}\right.$, Sigma), and $\mathrm{KCl}(1 \mathrm{M}$, Sigma) were mixed yielding a solution with the redox couple $\mathrm{Fe}^{2+} / \mathrm{Fe}^{3+}$ ions. The cyclic voltammograms that resulted from the electrochemical microchambers with the PPy-coated working electrode are shown in Figure 2.

Following the characterization, polypyrrole was deposited onto a copper template from a monomer solution. Polypyrrole was embedded in a flexible device configuration as described in our previous paper [6]. These devices can serve as sensors or actuators. Their structure consisted of metal (copper) connectors embedded on a flexible PDMS substrate. Polypyrrole was selectively deposited (using electropolymerization) on the copper layer as can be seen in Figure 3. Note that the windows in the passivation layer are misaligned with the contacts; nevertheless, full polypyrrole deposition was achieved.

Next we show the results of the PPy patterning by molding. The fabrication of patterned polypyrrole using molding technique was performed as well. Polypyrrole was deposited onto a gold template from a monomer solution. Selective electrochemical polymerization of polypyrrole was performed inside $1 \mu \mathrm{m}$ wide trenches patterned on $4-5 \mu \mathrm{m}$ thick photodefinable polymer (SU-8) on sputtered copper (or gold) on oxidized silicon (Figure 4). The relatively thick polypyrrole on the relatively thin metal seed may serve

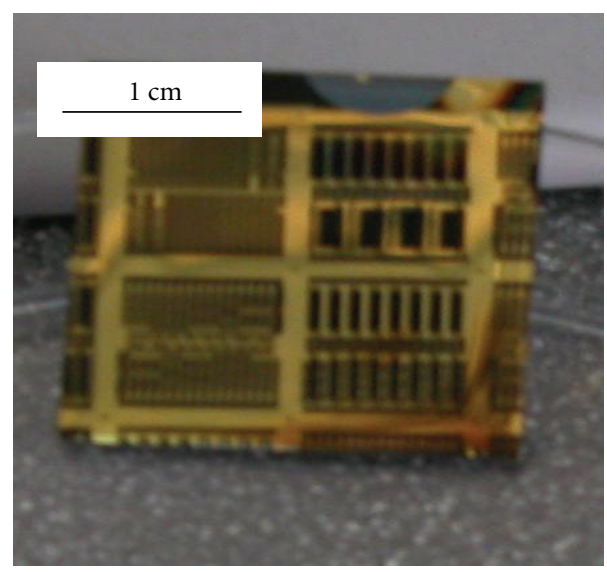

FIGURE 4: Optical micrograph of polypyrrole pattern with gold seed layer and SU-8 used a molding material.

as a compliant electrode allowing large deflections under electrostatic actuation of future fully polymeric actuators.

The thickness of the polypyrrole was measured using a Profilometer Tencor Alpha Step 500. The thickness of the polypyrrole as a function of the number of cycles is demonstrated in Figure 5. It can be seen that the thickness increased as the number of CV cycles increased.

When the potential is scanned from 0 to $1.2 \mathrm{~V}$ (the forward cycle) the anodic current density increases as an oxidized form of pyrrole is formed. When the potential is scanned from 1.1 to $0 \mathrm{~V}$ (the reverse cycle), considerably increased cathodic current appears, which suggests that a 


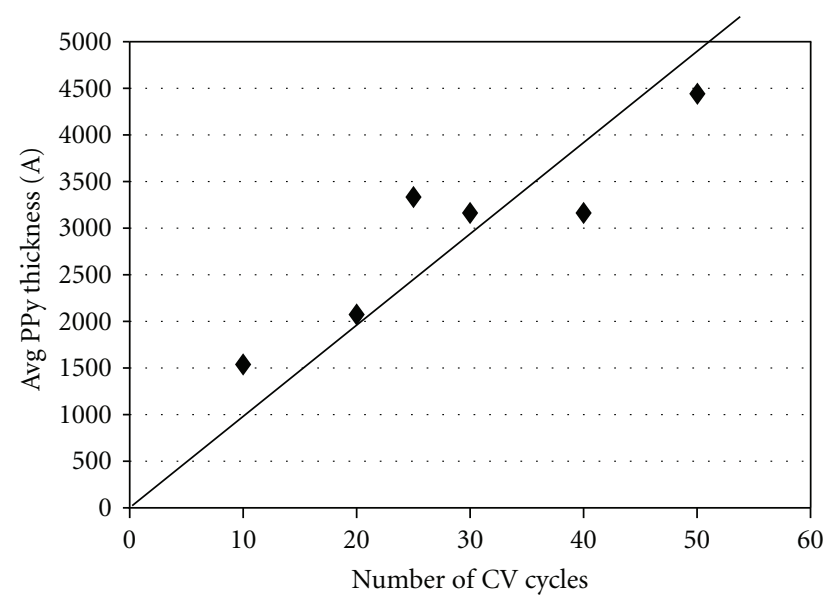

Figure 5: Thickness of the polypyrrole films, obtained by anodic electropolymerization of pyrrole as a function of the number of cycles.

cathodic process takes place. The oxidized form rehabilitates itself and therefore leads to a cathodic current. In the next forward cycles, as the amount of oxidized pyrrole increases, the cathodic current density increases again.

During the reverse cycle some of the oxidized pyrrole dissolves into the solution. However, we found that if the potential is kept in the interval of $0.3-1.1 \mathrm{~V}$, the amount of oxidized pyrrole created during the forward cycle is greater than the amount dissolved during the reverse cycle. Therefore, the thickness of the polypyrrole film increases with the number of cycles until saturation.

3.1. Electrochemical Characterization. The electrochemical activity of the electrode that was modified by PPy-coated working electrode was validated by a cyclic voltammetry assay with a redox couple of ferrocyanide/ferricyanide electroactive solution. The cyclic voltammograms that resulted from the electrochemical microchambers with the PPycoated working electrode are shown in Figure 2. The voltammograms using the electrochemical electrode show clearly the cathodic reduction of the $\mathrm{Fe}^{3+}$ and the anodic oxidation of the $\mathrm{Fe}^{2+}$. The current peak plot of both PPycoated and Au planar working electrodes (Figure 2) yielded a positive linear relation for anodic currents and a negative linear relation for cathodic currents.

The high currents, in the cyclic voltammograms of PPycoated electrode, in comparison to the cyclic voltammograms of flat gold electrode, indicate that the coating with PPy increases the effective surface area of the electrode.

We can assume that there is no electrochemical reactivity between the PPy and the solution, because the location of the reduction and oxidation picks in the cyclic voltammograms does not change with the addition of PPy to the gold electrode. Therefore, the redox potentials between the two electrodes stay the same.
The shape of the cyclic voltammograms with Ppy is similar to a rectangle, which also hints that there is a capacitor effect due to the large surface area.

The electropolymerization step provides a self-aligned deposition process. By using this polypyrrole deposition technique on the flexible devices, the polypyrrole reinforces the gold or the copper wires and electrically shunts the interconnects, thereby increasing the device durability and reliability, especially under stress conditions such as multiple electrode bending.

\section{Summary and Conclusions}

We introduce here the development of a photolithography process, which integrates an organic substrate with conductive polymers for biosensor electrode manufacture. The modification of the working electrode by PPy coating increases the effective surface area of the electrode. This is suggested by the high currents in the cyclic voltammograms of PPy-coated electrode, in comparison to the flat gold electrode. Moreover, the rectangular shape of the graph with PPy hints that there is capacitor effect caused by the large surface area.

The thick polypyrrole on the relatively thin metal seed (e.g., copper or gold) is sufficiently flexible to serve as a compliant electrode, thus allowing large deflections under electrostatic actuation. Although the metal is very ductile, it may develop defects and cracks due to the very large actuation amplitude and stress. The use of compliant polypyrrole electrodes allows shunting of the cracks in the metal, therefore increasing the device durability and reliability.

Future extension of the research presented here may be far reaching in its medical applications. Interfacing biological materials with MEMS devices may make it possible for microactuators and other MEMS devices to act as implants. Such an application presents important healthcare potential.

\section{References}

[1] S. Butefisch, V. Seidemann, and S. Buttgenbach, "Fabrication and investigation of in-plane compliant SU8 structures for MEMS and their application to micro valves and micro grippers," Sensors and Actuators A, vol. 97-98, pp. 457-461, 2002.

[2] V. T. Srikar and S. M. Spearing, "Materials selection for microfabricated electrostatic actuators," Sensors and Actuators A, vol. 102, no. 3, pp. 279-285, 2003.

[3] M. Calleja, M. Nordström, M. Álvarez, J. Tamayo, L. M. Lechuga, and A. Boisen, "Highly sensitive polymer-based cantilever-sensors for DNA detection," Ultramicroscopy, vol. 105, no. 1-4, pp. 215-222, 2005.

[4] B. A. Weisenberg and D. L. Mooradian, "Hemocompatibility of materials used in microelectromechanical systems: platelet adhesion and morphology in vitro," Journal of Biomedical Materials Research, vol. 60, no. 2, pp. 283-291, 2002.

[5] N. K. Guimard, N. Gomez, and C. E. Schmidt, "Conducting polymers in biomedical engineering," Progress in Polymer Science, vol. 32, no. 8-9, pp. 876-921, 2007. 
[6] Y. Shacham-Diamand, S. Krylov, T. Shmilovich et al., "Metallization technologies and Strategies for Plastic Based Biochips, Sensors and Actuators for Healthcare and Medical Applications," ECS Transactions, vol. 23, no. 1, pp. 243-254, 2009. 

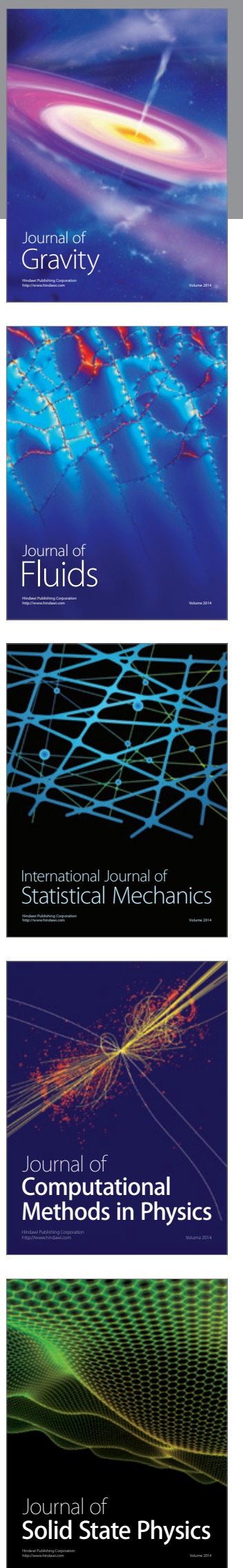

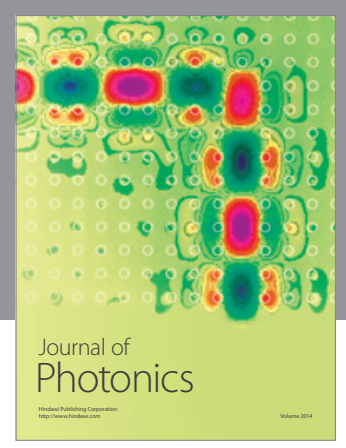

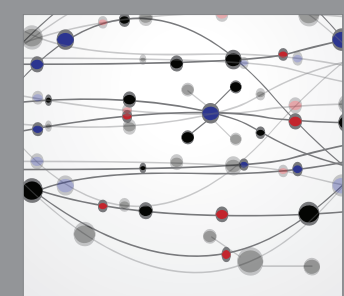

The Scientific World Journal
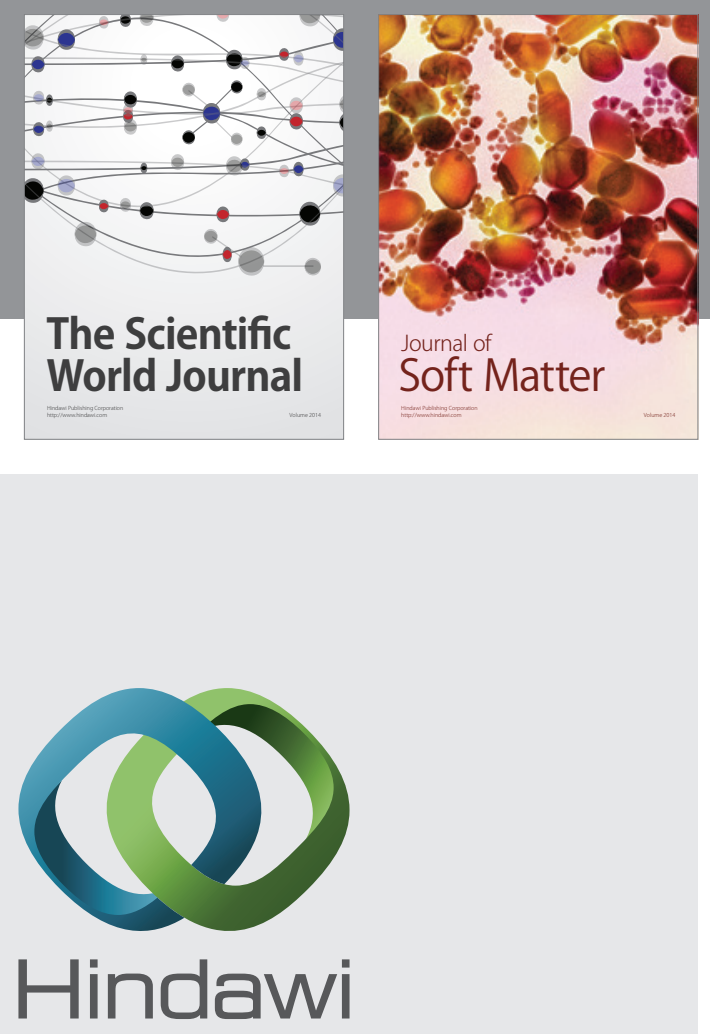

Submit your manuscripts at

http://www.hindawi.com
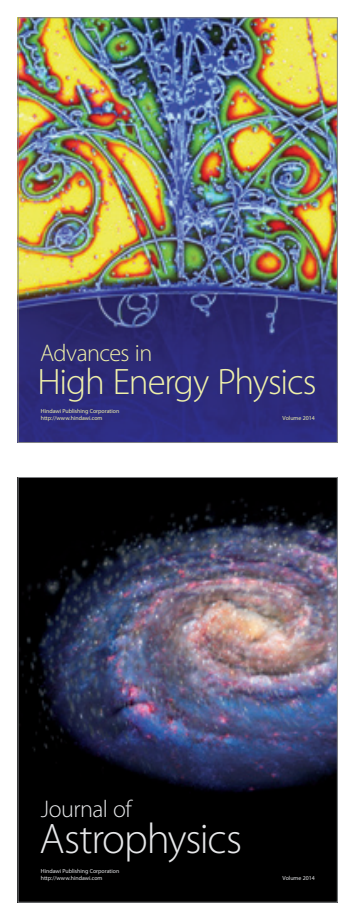
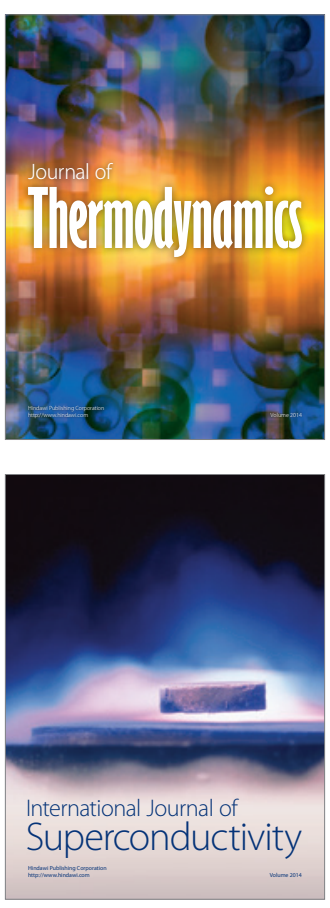
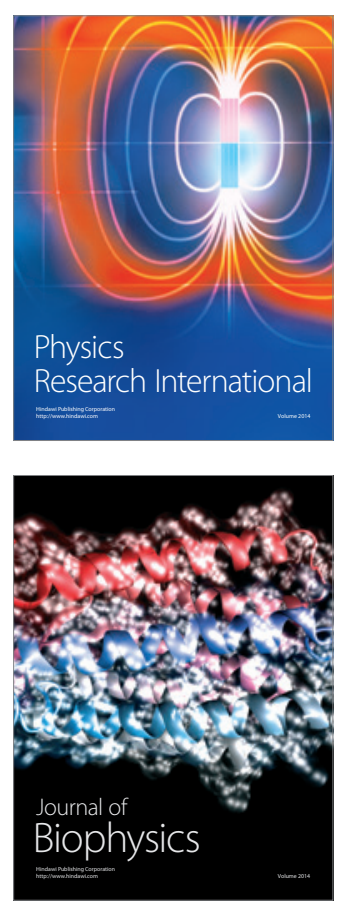
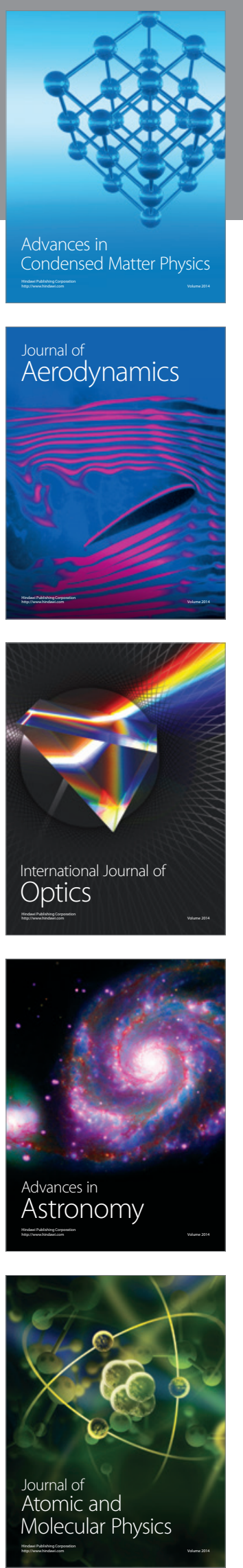